\title{
Uma discussão sobre o coeficiente de restituição
}

\author{
A discussion on coefficient of restitution
}

\author{
Damiao Pedro Meira Filho*1, Jorge Kysnney Santos Kamassury², Rose Caldas de Souza Meira ${ }^{3}$ \\ ${ }^{1}$ Instituto Federal do Pará, Santarém, PA, Brasil \\ ${ }^{2}$ Instituto de Engenharia e Geociências, Universidade Federal do Oeste do Pará, Santarém, PA, Brasil \\ ${ }^{3}$ Instituto de Ciências e Tecnologia das Águas, Universidade Federal do Oeste do Pará, Santarém, PA, Brasil
}

Recebido em 24 de Novembro de 2016. Revisado em 31 de Janeiro de 2017. Aceito em 26 de Fevereiro de 2017

\begin{abstract}
Seja em escala atômica, seja em escala macroscópica, a quantidade de eventos de colisão em nosso cotidiano é considerável e, portanto, os esforços em estudos que aumentam nossa compreensão destas colisões são essenciais tanto para o ensino como para o desenvolvimento de futuras tecnologias. Em processos de colisão, o coeficiente de restituição está associado ao grau de elasticidade da colisão entre os corpos em questão e nos permite classificar as colisões como elásticas, inelásticas e super-elásticas. No entanto, não é habitual observar na literatura uma abordagem matemática ampla e detalhada para explicar, de forma didática, esta classificação, o que acarreta o surgimento de dúvidas sobre o coeficiente de restituição. Nesse sentido, desenvolvemos neste artigo demonstrações detalhadas para cada caso, além de apresentar exemplos de colisões endoérgicas e colisões exoérgicas.
\end{abstract}

Palavras-chave: Coeficiente de restituição, colisões endoérgicas, colisões exoérgicas e tipos de colisão.

The amount of collision events in our daily lives, whether at the atomic scale or at macroscopic scale is considerable and therefore efforts in studies that increase our understanding of these collisions are essential both for teaching as for the development of future technologies. In collision processes, the coefficient of restitution is associated with the degree of elasticity of the collision between the bodies in concerned and allows us to classify the collision as elastic, inelastic and super-elastic. However, it is unusual to see in literature a comprehensive and detailed mathematical approach to explain, in a didactic way, this classification, which leads to the emergence of doubts about the coefficient of restitution. In accordance with this context, we develop in this article, detailed and appropriate demonstrations for each case, in addition to present examples of endoérgicas and exoérgicas collisions.

Keywords: Coefficient of restitution, endoergic collision, exoergic collision and types of collision.

\section{Introdução}

No estudo da dinâmica de um sistema de partículas, as colisões que ocorrem entre as partículas representam um dos tópicos mais relevantes na compreensão das interações físicas que ocorrem no sistema em questão.

Em foco, as colisões ocorrem quando a interação mútua de duas partículas (ou sistemas) que se aproximam alteram seus movimentos, produzindo, por consequência, uma troca de momento e energia. Em outras palavras, dizse que há colisão quando a aproximação entre partículas promove uma variação mensurável nos movimentos delas durante um curto intervalo de tempo (geralmente, desprezível em comparação ao tempo durante o qual se observa o sistema).

Seja na escala atômica, seja na escala macroscópica, a onipresença dos eventos de colisões no nosso cotidiano é significativa. Citam-se como exemplos de colisões, o choque entre duas bolas em uma mesa de bilhar, o chute de um jogador em uma bola de futebol, os acidentes entre veículos, as colisões entre partículas elementares em aceleradores de partículas, o choque de nêutrons com

*Endereço de correspondência: damiao.meira@ifpa.edu.br núcleos atômicos em um reator nuclear e até mesmo a colisão entre galáxias!

Umas das peculiaridades mais interessantes nesse campo de estudo incorre no fato de que, em geral, se conhecermos o movimento dos corpos antes da colisão ou depois da colisão, podemos obter informações relevantes como as características dos movimentos desses antes ou depois do choque entre eles. Especialistas em investigação de acidentes de tráfego, por exemplo, ao observarem detalhes acerca das trajetórias e do impacto de veículos que colidiram, conseguem fazendo o uso de conhecimentos sobre colisões e conservacão de momento, obter importantes informações sobre a velocidade e as direções do movimento dos veículos em instantes antes do choque. Um exemplo análogo ocorre nos grandes aceleradores de partícula como o LHC (Large Hadron Collider) e o FermiLab (Fermi National Accelerator Laboratory), nos quais, de posse dos dados computados antes e após as colisões, obtém-se, por exemplo, informações relativas às forças de interação no sistema que colide. As colisões promovidas nesses aceleradores são responsáveis por boa parte do conhecimento que temos acerca das forças fundamentais da natureza (excetuando nesse caso, a força 
gravitacional) e das propriedades e interações físicas das partículas elementares.

No estudo de colisões, costuma-se fazer uso do conceito de coeficiente de restituição $(e)$ que indica "o grau de elasticidade" da colisão [1]. Baseado nesse conceito, as colisões são classificadas como:

- $e=0$ : colisão perfeitamente inelástica;

- $0<e<1$ : colisão inelástica;

- $e=1$ : colisão perfeitamente elástica;

- $e>1$ : colisão superelástica;

Essa classificação é pertinente na dinâmica de sistemas de partículas, favorecendo uma compreensão dos tipos de colisões. Contudo, em geral, não se observa na literatura uma abordagem mais abrangente acerca da demonstração matemática e validade dessa classificação. Em outras palavras, a classificação é apresentada sem o devido desenvolvimento matemático e argumentação física sobre a mesma.

A ausência de uma discussão mais profunda dessa classificação pode suscitar perguntas interessantes; por exemplo, como demonstrar a impossibilidade da existência de $e<0$ ou mesmo demonstrar a existência de um $e>1$ ? A proposta desse artigo implica justamente em preencher essa lacuna na discussão do coeficiente de restituição, apresentando as demonstrações adequadas para cada caso.

\section{Conservação do Momento Linear e a Conservação da Energia}

A discussão apropriada do coeficiente de restituição exige a aplicação do conceito de conservação de momento linear e a conservação da energia do sistema em estudo. Portanto, para nortear a compreensão sobre a explanação física e matemática sobre a classificação das colisões a partir do coeficiente de restituição, segue nessa seção um resumo dos teoremas a serem utilizados.

\subsection{Momento linear de um sistema de partículas}

De imediato, o momento linear de um sistema de partículas é dado por (1), no qual, $\vec{p}_{i}, \vec{v}_{i}$ e $\vec{r}_{i}$ consistem, respectivamente, no momento linear, na velocidade e na posição (em relação a um referencial fixo) da partícula $i$.

$$
\vec{P}=\sum_{i=1} \vec{p}_{i}=\sum_{i=1} m_{i} \frac{d \vec{r}_{i}}{d t}
$$

Sabendo que o centro de massa de um sistema de partículas é dado por

$$
\vec{r}_{c m}=\frac{\sum_{i=1} m_{i} \vec{r}_{i}}{\sum_{i=1} m_{i}}
$$

e substituindo adequadamente (2) em (1), temos

$$
\begin{gathered}
\vec{P}=\sum_{i=1} m_{i} \frac{d \vec{r}_{i}}{d t}=\sum_{i=1} m_{i} \frac{d}{d t}\left(\frac{\sum_{i=1} m_{i} \vec{r}_{i}}{\sum_{i=1} m_{i}}\right)=M \vec{v}_{c m} \\
\vec{P}=M \vec{v}_{c m}
\end{gathered}
$$

onde $M=\sum_{i=1} m_{i}$ e $\vec{v}_{c m}$ é a velocidade do centro de massa do sistema. A relação (3) nos permite enunciar que o momento linear de um sistema de partículas é igual ao produto da massa total do sistema pela velocidade do centro de massa 2].

\subsection{Conservação do momento linear}

Consideremos um sistema de $N$ partículas e $\vec{F}_{i j}$ a força exercida pela partícula $i$ sobre a partícula $j$. Ademais, tomemos $\vec{F}_{i}^{\text {ext }}$ como a força externa total que atua sobre a partícula $i$.

De posse dessas considerações, a segunda lei de Newton para a i-ésima partícula é

$$
\frac{d \vec{p}_{i}}{d t}=m_{i} \frac{d^{2} \vec{r}_{i}}{d t^{2}}=\sum_{\substack{j=1 \\ i \neq j}}^{N} \vec{F}_{i j}+\vec{F}_{i}^{e x t}
$$

Avaliando o comportamento dinâmico de um sistema de $N$ partículas , obtemos a relação (4). Nesse contexto, vale mencionar que a validade de (4) decorre da hipótese de as massas das partículas serem invariantes temporalmente.

$$
\begin{gathered}
\sum_{i=1}^{N} \frac{d \vec{p}_{i}}{d t}=\frac{d}{d t} \sum_{i=1}^{N} \vec{p}_{i}=\sum_{i=1}^{N} \sum_{\substack{j=1 \\
i \neq j}}^{N} \vec{F}_{i j}+\sum_{i=1}^{N} \vec{F}_{i}^{e x t} \\
\frac{d \vec{P}}{d t}=\sum_{i=1}^{N} \sum_{\substack{j=1 \\
i \neq j}}^{N} \vec{F}_{i j}+\sum_{i=1}^{N} \vec{F}_{i}^{e x t}
\end{gathered}
$$

Do enunciado da terceira lei de Newton na sua forma forte, na qual, a força exercida pela partícula $i$ sobre a partícula $j$ é igual em módulo, porém em sentido contrário à força exercida pela partícula $j$ sobre a partícula $i$ e que tais forças são exercidas na direção que as une, temos:

$$
\vec{F}_{i j}=-\vec{F}_{j i}
$$

Podemos ainda utilizar (5) para alcançarmos a expressão 6.

$$
\begin{gathered}
\sum_{i=1}^{N} \sum_{\substack{j=1 \\
i \neq j}}^{N} \vec{F}_{i j}=\sum_{i=1}^{N} \sum_{\substack{j=1 \\
i \neq j}}^{N} \frac{1}{2}\left(\vec{F}_{i j}+\vec{F}_{j i}\right) \\
\sum_{i=1}^{N} \sum_{\substack{j=1 \\
i \neq j}}^{N} \vec{F}_{i j}=\overrightarrow{0}
\end{gathered}
$$


Desse modo, substituindo (6) em (4), obtemos

$$
\frac{d \vec{P}}{d t}=\sum_{i=1}^{N} \vec{F}_{i}^{e x t}
$$

que descrito em palavras, indica que a variação temporal do momento linear total de um sistema de partículas é igual a resultante das forças externas que atuam sobre esse sistema 3 .

O princípio da conservação do momento linear de um sistema decorre da relação (7) e enuncia que se a força externa total agindo sobre o sistema de partículas é nula, então

$$
\frac{d \vec{P}}{d t}=\overrightarrow{0}
$$

e, por conseguinte, o momento linear total do sistema permanece constante. Em particular, isso sempre vale na ausência de forças externas (um sistema isolado).

\subsection{Conservação da energia mecânica para um sistema de partículas}

De início, consideremos (9) o trabalho realizado por todas as forças que atuam num sistema de $N$ partículas (de massas constantes) para levá-lo de uma determinada configuração $a$ até uma outra configuração $b$, com $a \neq b$.

$$
W_{a b}=\sum_{i=1}^{N} \int_{a}^{b} \vec{F}_{i} \cdot d \vec{r}_{i}
$$

Ademais, consideremos as relações (10) e 11.

$$
\begin{gathered}
\vec{F}_{i} \cdot d \vec{r}_{i}=m_{i} \frac{d \vec{v}_{i}}{d t} \vec{v}_{i} d t \\
\frac{d}{d t}\left(\vec{v}_{i} \vec{v}_{i}\right)=\frac{d}{d t}\left|\vec{v}_{i}\right|^{2}=2 \vec{v}_{i} \frac{d \vec{v}_{i}}{d t} .
\end{gathered}
$$

Fazendo uso das relações (10) e (11), alcançamos a expressão 12

$$
\begin{gathered}
W_{a b}=\sum_{i=1}^{N} \frac{m_{i}}{2} \int_{a}^{b} d\left|\vec{v}_{i}\right|^{2}=\left.\sum_{i=1}^{N} \frac{m_{i}}{2}\left|\vec{v}_{i}\right|^{2}\right|_{a} ^{b} \\
W_{a b}=K_{b}-K_{a}
\end{gathered}
$$

onde, $K=\sum_{i=1}^{N} \frac{m_{i}}{2}\left|\vec{v}_{i}\right|^{2}$ representa a energia cinética de um sistema de partículas.

Deve-se mencionar que a expressão 12 é válida independente da natureza das forças que atuam sobre o sistema.
Se $\vec{F}_{i}$ possuir a propriedade em que $\int_{a}^{b} \vec{F}_{i} \cdot d \vec{r}_{i}$ independe do caminho, ou seja, $\int_{a}^{b} \vec{F}_{i} \cdot d \vec{r}_{i}$ tem o mesmo valor, qualquer que seja o caminho selecionado para levar um sistema de partículas de uma configuração $a$ para uma configuração $b$, então, nesse caso

$$
W_{a b}=-\sum_{i=1}^{N} \int_{a}^{b} \vec{F}_{i} \cdot d \vec{r}_{i}=-\left(V_{b}-V_{a}\right)
$$

na qual, $V$ representa a energia potencial do sistema para uma dada configuração e pode ser expressa como

$$
V=\sum_{i=1}^{N} V_{i}^{(e)}+\frac{1}{2} \sum_{\substack{j=1 \\ i \neq j}}^{N} V_{i j}
$$

Na expressão 14, $\sum_{i=1}^{N} V_{i}^{(e)}$ consiste na energia potencial decorrente das interações externas e $\frac{1}{2} \sum_{\substack{j=1 \\ i \neq j}}^{N} V_{i j}$, refere-se a energia potencial devido às interações internas das partículas do sistema 44.

Combinando as equações 12 e 13 , obtemos a energia mecânica $E$ de um sistema de partículas para uma determinada configuração, que, por sua vez, é constante quando só forças conservativas atual no sistema de estudo. Em essência, para uma dada configuração, a energia mecânica do sistema é

$$
E=\sum_{i=1}^{N}\left(\frac{m_{i}}{2}\left|\vec{v}_{i}\right|^{2}+V_{i}^{(e)}+\frac{1}{2} \sum_{\substack{j=1 \\ i \neq j}}^{N} V_{i j}\right)
$$

\section{A quantidade $\mathrm{Q}$}

Em um evento de colisão entre partículas, dependendo das forças atuantes, alguns resultados do processo são possíveis. Se as únicas forças atuantes no sistema de partículas forem as internas, então, a aplicação dos conceitos de conservação da energia e do momento linear dos sistemas de partículas é apropriado para uma abordagem acerca das características dessas colisões. Em muitos casos, a conservação do momento linear pode ser aplicada mesmo para sistemas que não sejam realmente isolados. Para tanto, a resultante das forças externas que atuam no sistema devem ser muito menor em comparação com as forças internas durante a colisão real [5].

Consideremos um processo de colisão entre duas partículas, sendo $p_{1}$ e $p_{2}$, os momentos lineares das partículas antes da colisão e $p_{1}^{\prime}$ e $p_{2}^{\prime}$, os momentos lineares dessas partículas após a colisão. Deve-se atentar que o uso do símbolo (') é empregado para indicar que a variável a qual ele está associada está numa condição pós-colisão. Por exemplo, $v^{\prime}$ indica a velocidade da partícula após a colisão. 
Portanto, não se deve confundir (') como sinônimo de operador diferencial.

Conforme a conservação do momento linear do sistema, podemos avaliar que

$$
p_{1}+p_{2}=p_{1}^{\prime}+p_{2}^{\prime}
$$

Consideremos ainda que a energia potencial e a energia cinética do sistema na, condição inicial, sejam $V_{(12)}$ e $K_{(12)}$ respectivamente, e que, por sua vez, $V_{(12)}^{\prime}$ e $K_{(12)}^{\prime}$ sejam a energia potencial e a energia cinética do sistema após a colisão, também respectivamente.

Nesse caso, segundo a conservação da energia do sistema de partículas, temos que

$$
K_{(12)}+V_{(12)}=K_{(12)}^{\prime}+V_{(12)}^{\prime}
$$

Nesse ínterim, convém introduzir a quantidade $Q$ que corresponde à diferença entre as energias cinéticas inicial e final do sistema ou mesmo pode ser definido como a diferença entre as energias potenciais internas final e inicial do referido sistema. Para a discussão seguinte, utilizamos essa última definição. A partir de agora, por motivos didáticos, usaremos $v^{2}$ para representar $|\vec{v}|^{2}$.

$$
Q=K_{(12)}-K_{(12)}^{\prime}=V_{(12)}^{\prime}-V_{(12)}
$$

Fazendo o uso de $Q$, podemos reescrever a relação 17 como

$$
\frac{1}{2} m_{1} v_{1}^{2}+\frac{1}{2} m_{2} v_{2}^{2}=\frac{1}{2} m_{1} v_{1}^{\prime 2}+\frac{1}{2} m_{2} v_{2}^{\prime 2}+Q
$$

A quantidade $Q$ presente na relação 19 pode ser:

- $Q>0$ : ocorre um decréscimo na energia cinética do sistema com acréscimo equivalente na energia potencial interna do mesmo. Nesse caso, temos uma colisão inelástica de primeira espécie (ou endoérgica);

- $Q=0$ : não ocorre variação na energia cinética do sistema (colisão elástica);

- $Q<0$ : ocorre um acréscimo na energia cinética do sistema devido ao decréscimo da energia potencial interna. Nesse caso, temos uma colisão inelástica de segunda espécie (ou exoérgica).

\section{Definição do coeficiente de restituição}

O coeficiente de restituição consiste na medida de elasticidade de uma colisão. Para uma colisão entre duas partículas é definido como

$$
e=\frac{\left|\vec{v}_{2}^{\prime}-\vec{v}_{1}^{\prime}\right|}{\left|\vec{v}_{2}-\vec{v}_{1}\right|}
$$

onde:

- $\left|\vec{v}_{2}-\vec{v}_{1}\right|$ representa o módulo da velocidade relativa de aproximação das partículas antes da colisão;

- $\left|\vec{v}_{2}^{\prime}-\vec{v}_{1}^{\prime}\right|$ representa o módulo da velocidade relativa de afastamento (recessão) das partículas após a colisão.

Para o caso unidimensional, é possível escrever

$$
e=\frac{\left|v_{2}^{\prime}-v_{1}^{\prime}\right|}{\left|v_{2}-v_{1}\right|}, \quad\left|v_{2}-v_{1}\right| \neq 0 .
$$

O coeficiente de restituição $e$ também é chamado de regra de Newton, uma vez, que o referido cientista descobriu experimentalmente que a proporção entre as velocidades relativas finais e as velocidades relativas iniciais no processo das colisões era aproximadamente constante para quaisquer dois corpos [6].

\subsection{Inversão temporal}

Se admitirmos que $\left|\vec{v}_{2}^{\prime}-\vec{v}_{1}^{\prime}\right| \neq 0$ e $\left|\vec{v}_{2}-\vec{v}_{1}\right| \neq 0$, então

$$
e_{(t)}=\frac{\left|v_{2}^{\prime}-v_{1}^{\prime}\right|}{\left|v_{2}-v_{1}\right|}=\frac{\left|\frac{d r_{2}^{\prime}}{d t}-\frac{d r_{1}^{\prime}}{d t}\right|}{\left|\frac{d r_{2}}{d t}-\frac{d r_{1}}{d t}\right|} .
$$

Assumindo ainda que o processo de colisão decorre no sentido inverso $(t \rightarrow-t)$, verificamos:

- $\left|\vec{v}_{2}-\vec{v}_{1}\right|$ representa o módulo da velocidade relativa de afastamento das partículas antes da colisão;

- $\left|\vec{v}_{2}^{\prime}-\vec{v}_{1}^{\prime}\right|$ representa o módulo da velocidade relativa de aproximação das partículas após a colisão.

Com base nessas considerações, podemos alcançar a relação $(23)$

$$
\begin{gathered}
e_{(-t)}=\frac{\left|v_{2}-v_{1}\right|}{\left|v_{2}^{\prime}-v_{1}^{\prime}\right|}=\frac{\left|\frac{d r_{2}}{d(-t)}-\frac{d r_{1}}{d(-t)}\right|}{\left|\frac{d r_{2}^{\prime}}{d(-t)}-\frac{d r_{1}^{\prime}}{d(-t)}\right|} \\
e_{(-t)}=\frac{\left|-\left(\frac{d r_{2}}{d t}-\frac{d r_{1}}{d t}\right)\right|}{\left|-\left(\frac{d r_{2}^{\prime}}{d t}-\frac{d r_{1}^{\prime}}{d t}\right)\right|} \\
e_{(-t)}=\frac{\left|\frac{d r_{2}}{d t}-\frac{d r_{1}}{d t}\right|}{\left|\frac{d r_{2}^{\prime}}{d t}-\frac{d r_{1}^{\prime}}{d t}\right|} .
\end{gathered}
$$

Por fim, comparando 22 e (23), obtemos

$$
e_{(t)}=\frac{1}{e_{(-t)}}
$$




\subsection{Classificação das colisões segundo o coeficiente de restituição}

Fazendo uso do conceito de coeficiente de restituição, as colisões podem ser elencadas como:

- $e=0$ : colisão perfeitamente inelástica (a velocidade das partículas do sistema após a colisão são iguais. A energia cinética do sistema após a colisão é menor que a energia cinética antes da colisão);

- $0<e<1$ : colisão inelástica (a energia cinética do sistema após a colisão é menor que a energia cinética antes da colisão);

- $e=1$ : colisão perfeitamente elástica (a energia cinética do sistema após a colisão é igual a energia cinética antes da colisão);

- $e>1$ : colisão superelástica (a energia cinética do sistema após a colisão é maior que a energia cinética antes da colisão).

\section{Discussão física e matemática da classificação das colisões}

Em geral, a análise física e matemática das colisões elásticas já é bem discutida na literatura. Tão-logo, a discussão que se segue é desenvolvida para as colisões inelásticas (colisões endoérgicas e colisões exoérgicas).

\subsection{Colisões endoérgicas}

De imediato, para uma colisão endoérgica, a expressão (19) pode ser reescrita como

$$
\frac{1}{2} m_{1} v_{1}^{2}+\frac{1}{2} m_{2} v_{2}^{2}>\frac{1}{2} m_{1} v_{1}^{\prime 2}+\frac{1}{2} m_{2} v_{2}^{\prime 2} .
$$

Rerranjando (25), temos

$$
\begin{gathered}
m_{1} v_{1}^{2}+m_{2} v_{2}^{2}>m_{1}{v^{\prime}}_{1}^{2}+m_{2} v_{2}^{\prime 2} \\
m_{1}\left(v_{1}^{2}-v_{1}^{\prime 2}\right)>m_{2}\left(v_{2}^{\prime 2}-v_{2}^{2}\right) \\
m_{1}\left(v_{1}-v_{1}^{\prime}{ }_{1}\right)\left(v_{1}+v^{\prime}{ }_{1}\right)>m_{2}\left(v_{2}^{\prime}{ }_{2}-v_{2}\right)\left(v_{2}^{\prime}+v_{2}\right)
\end{gathered}
$$

Para esse sistema de duas partículas, a conservação do momento linear é dada por

$$
m_{1} v_{1}+m_{2} v_{2}=m_{1} v_{1}^{\prime}+m_{2} v_{2}^{\prime}
$$

Essa última expressão 27 nos permite obter

$$
m_{1}\left(v_{1}-v_{1}^{\prime}\right)=m_{2}\left(v_{2}^{\prime}-v_{2}\right)
$$

Assumindo que $\left(v_{1}-v_{1}^{\prime}\right)>0$, então, por consequência, $\left(v_{2}^{\prime}-v_{2}\right)>0$. Ao dividirmos o primeiro membro da equação (26) por $m_{1}\left(v_{1}-v_{1}^{\prime}\right)$ e o segundo membro por $m_{2}\left(v_{2}^{\prime}-v_{2}\right)$, verifica-se que

$$
\begin{aligned}
\frac{m_{1}\left(v_{1}-v_{1}^{\prime}\right)\left(v_{1}+v_{1}^{\prime}\right)}{m_{1}\left(v_{1}-v_{1}^{\prime}\right)}>\frac{m_{2}\left(v_{2}^{\prime}-v_{2}\right)\left(v_{2}^{\prime}+v_{2}\right)}{m_{2}\left(v_{2}^{\prime}-v_{2}\right)} & \\
v_{1}+v_{1}^{\prime} & >v_{2}^{\prime}+v_{2} \\
v_{1}-v_{2} & >v_{2}^{\prime}-v_{1}^{\prime} .
\end{aligned}
$$

\subsection{1. $1^{\mathrm{o}}$ Caso}

Com base na relação 29 , a primeira análise que pode ser realizada é:

$$
\left\{\begin{array}{l}
v_{1}-v_{2}>0 \\
v_{2}^{\prime}-v_{1}^{\prime}>0
\end{array}\right.
$$

Utilizando a definição de módulo, temos:

$$
\begin{gathered}
v_{1}-v_{2}=\left|v_{1}-v_{2}\right|=\left|-\left(v_{2}-v_{1}\right)\right|=\left|v_{2}-v_{1}\right| \\
v_{2}^{\prime}-v_{1}^{\prime}=\left|v_{2}^{\prime}-v_{1}^{\prime}\right|
\end{gathered}
$$

Substituindo essas observações na relação 29 e dividindo (29) por $\left|v_{2}-v_{1}\right|$, obtemos:

$$
\begin{aligned}
\left|v_{2}-v_{1}\right| & >\left|v_{2}^{\prime}-v_{1}^{\prime}\right| \\
\frac{\left|v_{2}-v_{1}\right|}{\left|v_{2}-v_{1}\right|}>\frac{\left|v_{2}^{\prime}-v_{1}^{\prime}\right|}{\left|v_{2}-v_{1}\right|} & \\
1 & >\frac{\left|v_{2}^{\prime}-v_{1}^{\prime}\right|}{\left|v_{2}-v_{1}\right|} \\
e & <1
\end{aligned}
$$

Desse modo, demonstra-se a possibilidade matemática do coeficiente de restituição ser menor que 1. As situações físicas possíveis para esse $1^{\mathrm{o}}$ caso (antes e após a colisão) estão ilustradas na figura (1). Por motivos didáticos, priorizou-se analisar os casos em que as velocidades das partículas antes e após a colisão são diferentes de zero.

\subsection{2. $2^{\mathrm{o}}$ Caso}

Novamente de posse da expressão (29), conduzimos nossa análise para o caso em que

$$
\left\{\begin{array}{l}
v_{1}-v_{2}>0 \\
v_{2}^{\prime}-v_{1}^{\prime}<0
\end{array}\right.
$$

Aplicando a relação de módulo, obtemos:

$$
\begin{gathered}
v_{1}-v_{2}=\left|v_{2}-v_{1}\right| \\
v_{2}^{\prime}-v_{1}^{\prime}=-\left|v_{2}^{\prime}-v_{1}^{\prime}\right|
\end{gathered}
$$


A

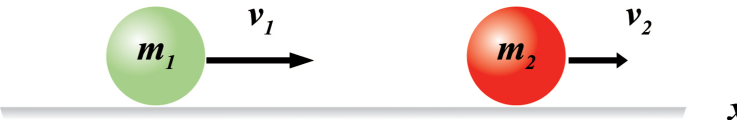

B

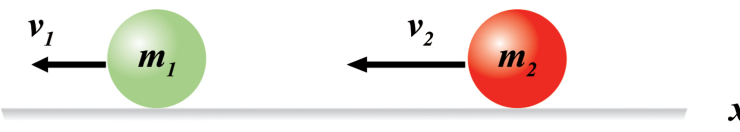

C

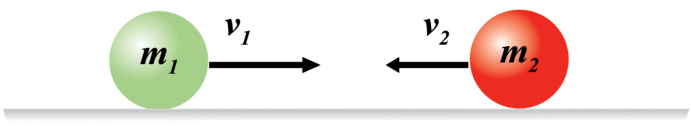

$\boldsymbol{x}$
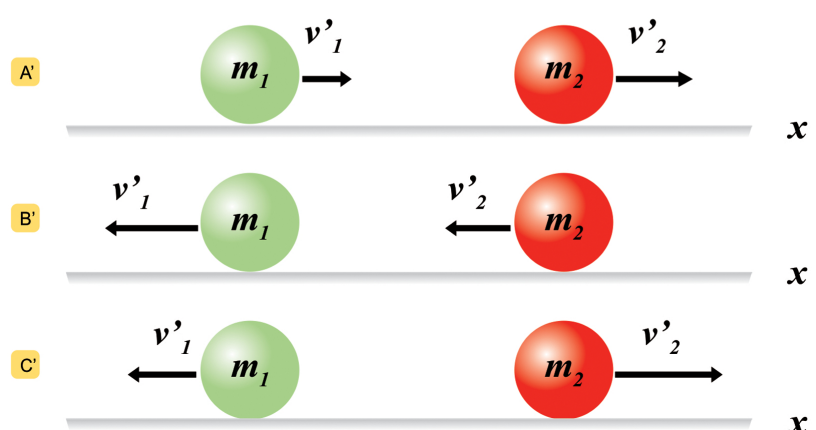

Figura 1: $v_{1}-v_{2}>0$ antes da colisão; $v_{2}^{\prime}-v_{1}^{\prime}>0$ depois da colisão (de cima para baixo).

Repetindo o procedimento realizado no caso anterior, alcançamos:

$$
\begin{gathered}
\frac{\left|v_{2}^{\prime}-v_{1}^{\prime}\right|}{\left|v_{2}-v_{1}\right|}>-\frac{\left|v_{2}-v_{1}\right|}{\left|v_{2}-v_{1}\right|} \\
e>-1
\end{gathered}
$$

Deve-se atentar que o resultado em (31) decorre de uma abordagem essencialmente matemática! Observa-se que para as situações nas quais $v_{1}-v_{2}>0$, a relação $v_{2}^{\prime}-v_{1}^{\prime}<0$ não descreve situações físicas reais após a colisão conforme ilustra a figura (2) e, desse modo, pode-se concluir que $e>-1$ não representa processos de colisão possíveis.

\subsection{3. $3^{\mathrm{o}}$ Caso}

Para esse caso, consideremos que

$$
\left\{\begin{array}{l}
v_{1}-v_{2}<0 \\
v_{2}^{\prime}-v_{1}^{\prime}<0
\end{array}\right.
$$

Nesse caso, temos:

$$
\begin{gathered}
v_{1}-v_{2}=-\left|v_{2}-v_{1}\right| \\
v_{2}^{\prime}-v_{1}^{\prime}=-\left|v_{2}^{\prime}-v_{1}^{\prime}\right| .
\end{gathered}
$$

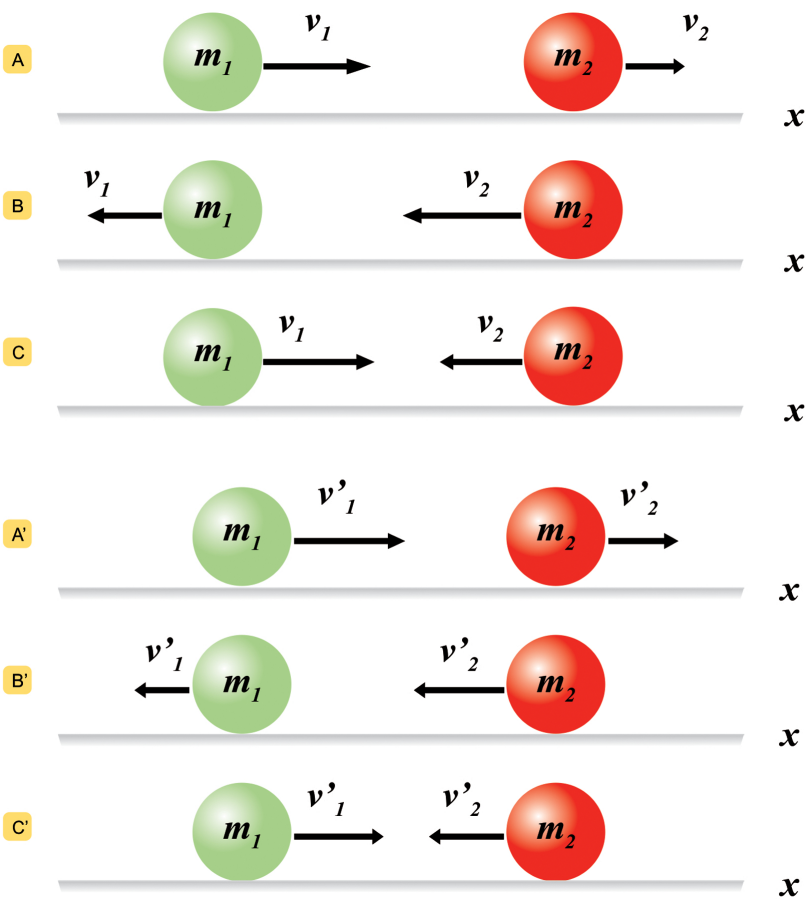

Figura 2: $v_{1}-v_{2}>0$ antes da colisão; $v_{2}^{\prime}-v_{1}^{\prime}<0$ depois da colisão (de cima para baixo). Nesse caso, $v_{2}^{\prime}-v_{1}^{\prime}<0$ não descreve situações físicas possíveis após a colisão.

Fazendo uso dessas últimas relações, verificamos que:

$$
\begin{aligned}
\frac{\left|v_{2}^{\prime}-v_{1}^{\prime}\right|}{\left|v_{2}-v_{1}\right|}>\frac{\left|v_{2}-v_{1}\right|}{\left|v_{2}-v_{1}\right|} \\
e>1 .
\end{aligned}
$$

Para avaliar o resultado em (32) há a necessidade de realizarmos algumas considerações. As condições inicias para o estudo da colisão endoérgica informam que

$$
\left\{\begin{array}{c}
v_{1}>v_{1}^{\prime} \\
v_{2}^{\prime}>v_{2} .
\end{array}\right.
$$

Fazendo uso da relação $v_{1}-v_{2}<0$, sabe-se que $v_{1}<v_{2}$. Uma vez que $v_{2}^{\prime}>v_{2}>v_{1}$, então $v_{2}^{\prime}>v_{1}$. Observando que $v_{1}>v_{1}^{\prime}$, então:

$$
v_{2}^{\prime}-v_{1}^{\prime}>0
$$

Nesse caso, percebe-se que (33) contradiz a relação inicial $v_{2}^{\prime}-v_{1}^{\prime}<0$. Portanto, o resultado 32 não descreve processos de colisões reais. Em outras palavras, conclui-se que para colisões endoérgicas, $e>1$ não se aplica.

\subsection{Colisões exoérgicas}

Sabe-se que para uma colisão exoérgica, a expressão (19) pode ser reescrita como

$$
\frac{1}{2} m_{1}{v_{1}^{\prime}}_{1}^{2}+\frac{1}{2} m_{2} v_{2}^{\prime 2}>\frac{1}{2} m_{1} v_{1}^{2}+\frac{1}{2} m_{2} v_{2}^{2} .
$$


Rerranjando (34), temos

$$
\begin{gathered}
m_{1} v_{1}^{\prime 2}+m_{2}{v_{2}^{\prime}}_{2}^{2}>m_{1} v_{1}^{2}+m_{2} v_{2}^{2} \\
m_{1}\left(v_{1}^{\prime 2}-v_{1}^{2}\right)>m_{2}\left(v_{2}^{2}-v_{2}^{\prime 2}\right) \\
m_{1}\left(v_{1}^{\prime}-v_{1}\right)\left(v_{1}^{\prime}+v_{1}\right)>m_{2}\left(v_{2}-v_{2}^{\prime}\right)\left(v_{2}+v_{2}^{\prime}\right) .
\end{gathered}
$$

Podemos reescrever a conservação do momento linear e obter

$$
-m_{1}\left(v_{1}^{\prime}-v_{1}\right)=-m_{2}\left(v_{2}-v_{2}^{\prime}\right)
$$

Considerando que $\left(v_{1}^{\prime}-v_{1}\right)<0$, então, $\left(v_{2}-v_{2}^{\prime}\right)<0$. Se dividirmos o primeiro membro da equação (35) por $-m_{1}\left(v_{1}^{\prime}-v_{1}\right)$ e o segundo membro por $-m_{2}\left(v_{2}-v_{2}^{\prime}\right)$, temos

$$
\begin{gathered}
\frac{m_{1}\left(v_{1}^{\prime}-v_{1}\right)\left(v_{1}^{\prime}+v_{1}\right)}{m_{1}\left(v_{1}^{\prime}-v_{1}\right)}<\frac{m_{2}\left(v_{2}-v_{2}^{\prime}\right)\left(v_{2}+v_{2}^{\prime}\right)}{m_{2}\left(v_{2}-v_{2}^{\prime}\right)} \\
v_{1}^{\prime}+v_{1}<v_{2}+v_{2}^{\prime} \\
v_{1}-v_{2}<v_{2}^{\prime}-v_{1}^{\prime} .
\end{gathered}
$$

\subsection{1. $1^{\mathrm{O}}$ Caso}

De início, consideremos que:

$$
\left\{\begin{array}{l}
v_{1}-v_{2}>0 \\
v_{2}^{\prime}-v_{1}^{\prime}>0
\end{array}\right.
$$

Sabendo que

$$
\begin{aligned}
& v_{1}-v_{2}=\left|v_{2}-v_{1}\right| \\
& v_{2}^{\prime}-v_{1}^{\prime}=\left|v_{2}^{\prime}-v_{1}^{\prime}\right|
\end{aligned}
$$

e substituindo essas relações em (37) e dividindo (37) por $\left|v_{2}-v_{1}\right|$, obtemos:

$$
\begin{aligned}
\left|v_{2}-v_{1}\right| & <\left|v_{2}^{\prime}-v_{1}^{\prime}\right| \\
1 & <\frac{\left|v_{2}^{\prime}-v_{1}^{\prime}\right|}{\left|v_{2}-v_{1}\right|} \\
e & >1
\end{aligned}
$$

O resultado em (38) demonstra que para colisões exoérgicas, o coeficiente de restituição pode ser maior que 1 (colisão superelástica). Nesse contexto, as situações físicas possíveis estão ilustradas na figura (3).

\subsection{2. $2^{\mathrm{o}}$ Caso}

Analisemos o caso em que

$$
\left\{\begin{array}{l}
v_{1}-v_{2}<0 \\
v_{2}^{\prime}-v_{1}^{\prime}>0
\end{array}\right.
$$

A

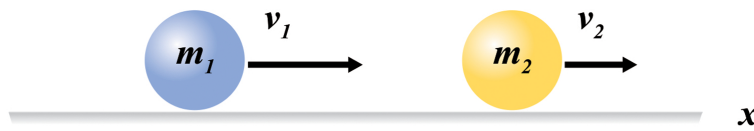

B

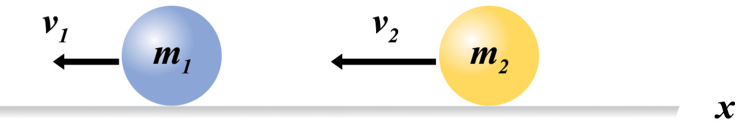

C

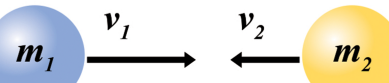

$A^{\prime}$

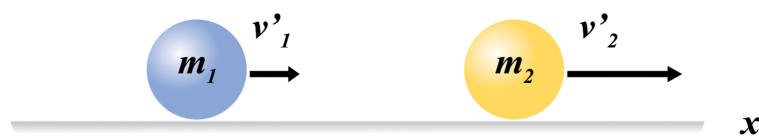

$B^{\prime}$

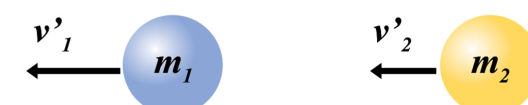

C

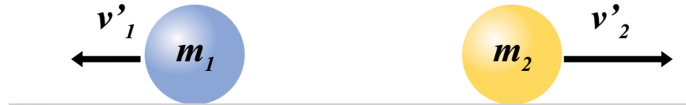

$\boldsymbol{x}$

Figura 3: $v_{1}-v_{2}>0$ antes da colisão; $v_{2}^{\prime}-v_{1}^{\prime}>0$ após a colisão (de cima para baixo). Nesses casos, o coeficiente de restituição é maior que 1 .

De posse das seguintes relações

$$
\begin{array}{r}
v_{1}-v_{2}=-\left|v_{2}-v_{1}\right| \\
v_{2}^{\prime}-v_{1}^{\prime}=\left|v_{2}^{\prime}-v_{1}^{\prime}\right|
\end{array}
$$

e repetindo o procedimento realizado no $1^{\mathrm{O}}$ caso das colisões exoérgicas, alcançamos :

$$
\begin{gathered}
-1<\frac{\left|v_{2}^{\prime}-v_{1}^{\prime}\right|}{\left|v_{2}-v_{1}\right|} \\
e>-1
\end{gathered}
$$

Embora esse caso nos retorne um $e>-1$, vale observar que para colisões exoérgicas a relação $v_{2}^{\prime}-v_{1}^{\prime}>0$ não descreve situações físicas possíveis. A figura (4) nos evidencia tal impossibilidade.

\subsection{3. $3^{\mathrm{o}}$ Caso}

Por fim, consideremos o caso em que

$$
\left\{\begin{array}{l}
v_{1}-v_{2}<0 \\
v_{2}^{\prime}-v_{1}^{\prime}<0
\end{array}\right.
$$

Observe que para esse caso:

$$
\begin{gathered}
v_{1}-v_{2}=-\left|v_{2}-v_{1}\right| \\
v_{2}^{\prime}-v_{1}^{\prime}=-\left|v_{2}^{\prime}-v_{1}^{\prime}\right| .
\end{gathered}
$$




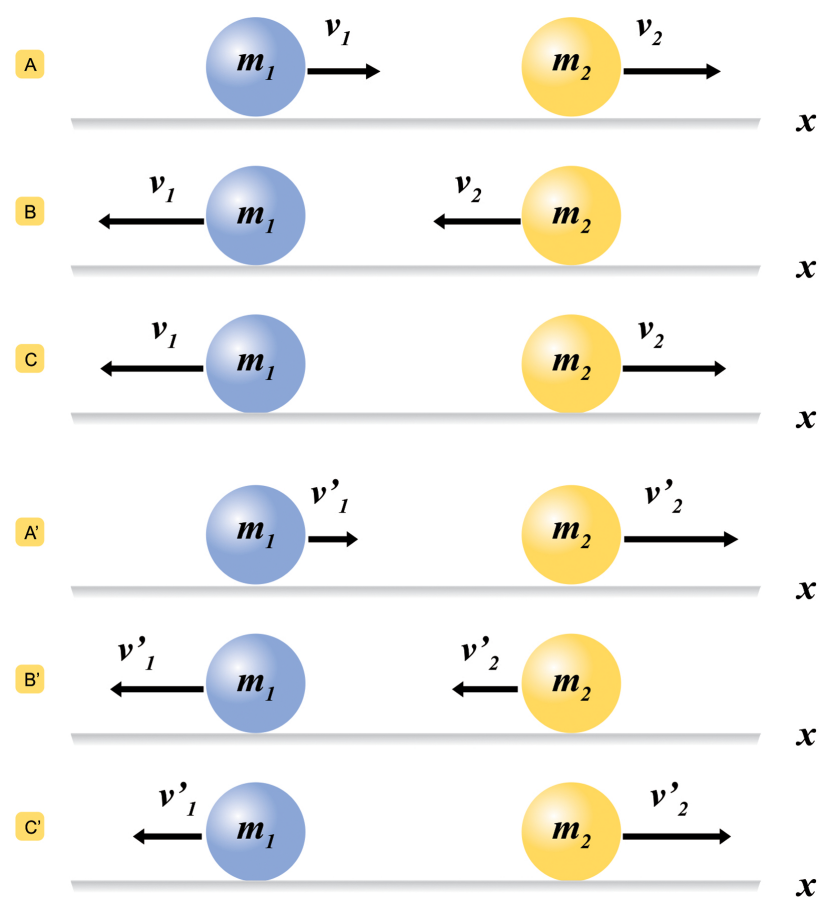

Figura 4: $v_{1}-v_{2}<0$ antes da colisão; $v_{2}^{\prime}-v_{1}^{\prime}>0$ após a colisão (de cima para baixo). Para as colisões exoérgicas, essas situações pós-colisão são impossíveis.

Utilizando essas últimas relações, facilmente verificamos que:

$$
\begin{aligned}
& e<\frac{\left|v_{2}-v_{1}\right|}{\left|v_{2}-v_{1}\right|} \\
& e<1 .
\end{aligned}
$$

Esse resultado para o coeficiente de restituição exige relevantes observações. De início, as condições iniciais utilizadas na análise das colisões exoérgicas enunciam que

$$
\left\{\begin{array}{l}
v_{1}>v_{1}^{\prime} \\
v_{2}^{\prime}>v_{2} .
\end{array}\right.
$$

Contudo, para esse caso, sabe-se que $v_{1}-v_{2}<0$. Além disso, uma vez que $v_{2}^{\prime}>v_{2}>v_{1}$, por consequência, $v_{2}^{\prime}>v_{1}$. Ora, se $v_{1}>v_{1}^{\prime}$, pode-se afirmar que $v_{2}^{\prime}>v_{1}^{\prime}$, e, portanto que

$$
v_{2}^{\prime}-v_{1}^{\prime}>0
$$

Todavia,a relação 41 é incompatível com a expressão inicial desse caso $\left(v_{2}^{\prime}-v_{1}^{\prime}<0\right)$. Com base nessa argumentação, pode-se avaliar que $e<1$ não descreve situações físicas reais para colisões exoérgicas.

\section{Exemplos e aplicações}

\subsection{Colisões endoérgicas ou colisões inelásticas de primeira espécie}

São reconhecidamente as colisões mais frequentes no nosso cotidiano, sendo caracterizadas pela redução da energia cinética do sistema após o evento de colisão. Nesse caso, parte da energia cinética antes da colisão é convertida em outras formas de energia como a térmica, a luminosa, a sonora e a cinética vibracional ou, até mesmo, investida na realização de trabalho de deformação permanente. As colisões endoérgicas representam quase a totalidade dos eventos de colisões entre corpos macrocóspicos. Como exemplos, citam-se os acidentes entre carros, o choque de asteróides com planetas, as colisões das moléculas de um fluido com as paredes de um recipiente e a movimentação das pás de turbinas através da ação de correntes de fluido ( figura 5).

Nos reatores nucleares esse tipo de colisão é estrategicamente necessário para o processo de moderação de neutrôns. Os núcleos de $U^{235}$, por exemplo, capturam nêutrons e sofrem fissão nuclear, liberando energia e emitindo nêutrons rápidos que possuem energia média da ordem de $1 \mathrm{MeV}$. Para viabilizar uma eficiente reação em cadeia (novas fissões), essas partículas devem ter suas velocidades reduzidas (moderadas) às velocidades de equilíbrio térmico 3 mediante colisões com específicas partículas-alvo. A escolha dessas partículas-alvo está ancorada fudamentalmente nos conhecimentos relativos às informações pós-colisão entre corpos com massas iguais ou distintas.

O estudo de colisões prevê, por exemplo, que quando uma particula muito leve e que está em repouso é atingida por uma partícula pesada, está última quase não é freiada, enquanto que a mais leve sai do repouso com aproximadamente o dobro da velocidade da partícula incidente. Esse conhecimento justifica o fato de não serem empregadas partículas como os elétrons para moderar os referidos nêutrons, afinal, além de não freiar os nêutrons,

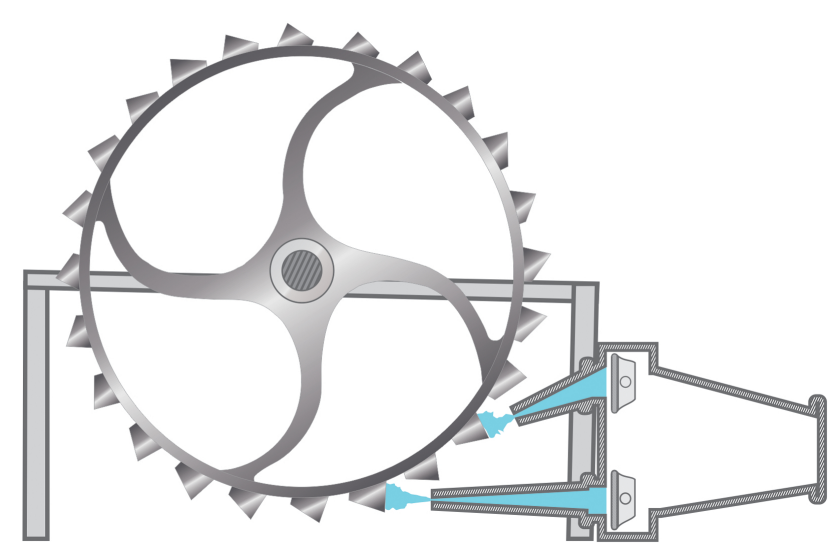

Figura 5: Vista lateral simplificada de uma turbina do tipo Pelton que converte a energia de pressão do escoamento em energia cinética. 


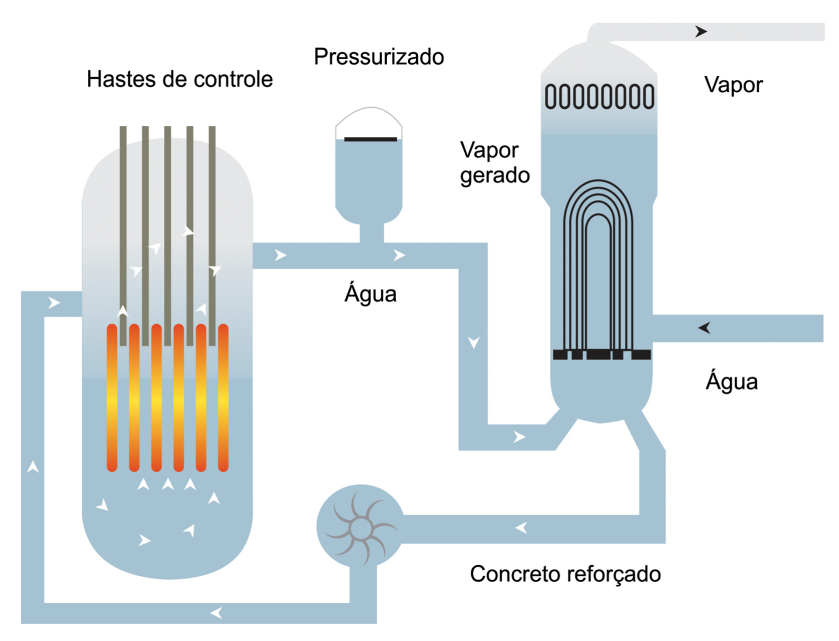

Figura 6: Esquema de funcionamento de um reator de água leve pressurizada.

os elétrons seriam acelerados. Por outro lado, se os alvos estacionários forem partículas de massas aproximadamente iguais às massa dos nêutrons, como o próton, é previsto que essas partículas incidentes após a colisão ficarão praticamente em repouso. De posse dessa análise, o hidrogênio que possui a mesma massa que o próton seria a partícula-alvo ideal para o processo de moderação; entretanto, esse elemento captura nêutrons lentos, inviabilizando desse modo o ciclo de fissão. Logo, os moderadores ideiais são aquelas partículas leves e que não capturam exarcerbadamente nêutrons 3. Atualmente, as tecnologias associadas aos reatores nuncleares utilizam como moderadores a água leve (como é o caso das usinas nucleares 1 e 2 de Angra dos Reis no Brasil), o deutério (presente na água pesada), o berílio e o carbono (na forma de grafite ou parafina).

\subsubsection{Colisões perfeitamente inelásticas}

Um caso especial da classe de colisões endoérgicas são as colisões perfeitamente elásticas, nas quais, ocorre a máxima redução de energia cinética possível após o choque entre as partículas. Uma vez que a energia cinética do sistema está associada ao movimento do centro de massa, a máxima redução dessa energia ocorre quando não há movimento relativo das partículas em relação ao centro de massa. Essa configuração indica que as partículas têm de se mover juntas após a colisão. Uma bola de argila que atinge o piso e não ricocheteia, um dardo que atinge seu alvo, um peixe que engole sua presa, a colisão suave entre um vagão ferroviário em movimento e um outro vagão inicialmente em repouso e até mesmo acoplamentos de naves em estações espaciais são exemplos de colisões perfeitamente inelásticas (figura 7).

Nos laboratórios de ensino, uma das experiências mais empregadas para o estudo das colisões perfeitamente inelásticas consiste no pêndulo balístico. Idealizado e projetado em 1742 pelo matemático e engenheiro militar inglês Benjamin Robins (1707-1752), o pêndulo balístico é

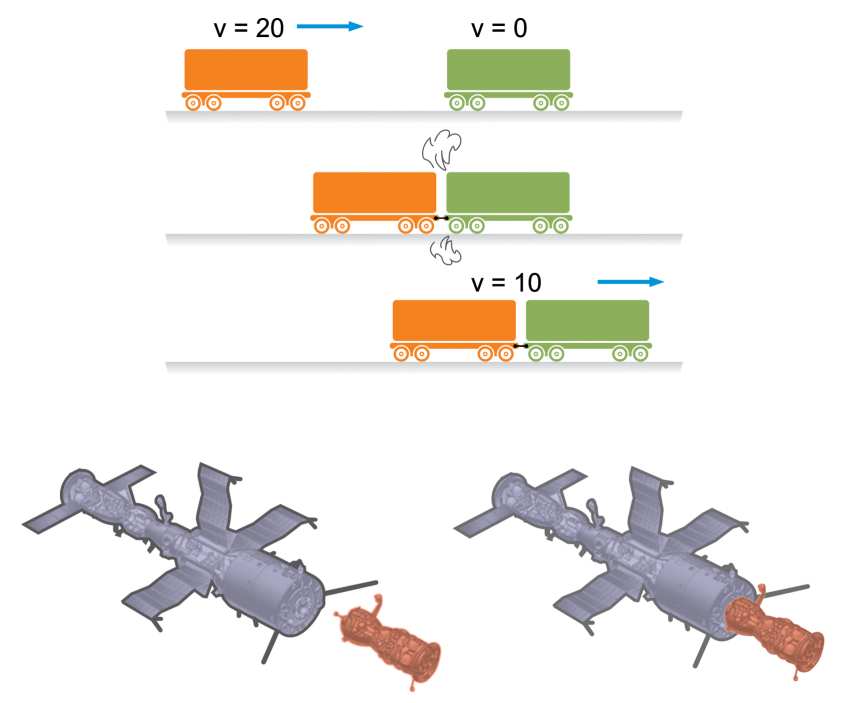

Figura 7: Exemplos de colisões perfeitamente inelásticas: acoplamento entre vagões ferroviários e acoplamento entre nave e estação espacial (de cima para baixo).

um dispositivo capaz de medir a velocidade de projéteis de armas de fogo [7]. Embora existam diversas configurações desse experimento, pode-se facilmente compreender o funcionamento do mesmo pelo esquema dado pela figura (8).

Em destaque, o pêndulo consiste de um grande bloco de madeira suspenso verticamente por fios. Quando um projétil é disparado com um determinada velocidade inicial contra esse bloco de madeira, o mesmo aloja-se neste e tal bloco sobe a uma altura de oscilação máxima. Uma vez que o tempo de colisão é muito inferior quando comparado com o período de oscilação, podemos admitir que os fios que sustentam o pêndulo permanecem praticamente na vertical durante o evento de colisão, o que nos permite tratar o evento como um processo unidimensional. Empregando adequadamente a conservação do momento linear e a conservação da energia mecânica, pode-se calcular a velocidade inicial do projétil.

\subsection{Colisões elásticas}

Caracterizada pela conservação da energia cinética do sistema, essas colisões que possuem coeficiente de restituição igual a unidade são relativamente comuns na escala microscópica, como é o caso daquelas que ocorrem entre moléculas de ar às temperaturas encontradas na superfície da Terra [8]. De fato, as colisões no nível microscópico são as únicas realmente elásticas conhecidas, uma vez, que aquelas resultantes das interações entre corpos macroscópicos são inelásticas em algum grau. Entretanto, em algumas situações, podemos tratá-las como se fossem elásticas! O choque entre duas bolas de bilhar, por exemplo, é inegavelmente um caso de colisão inelástica em que parte da energia mecânica do sistema é convertida em energia sonora e energia térmica. Contudo, a perda total de energia cinética é relativamente pequena 


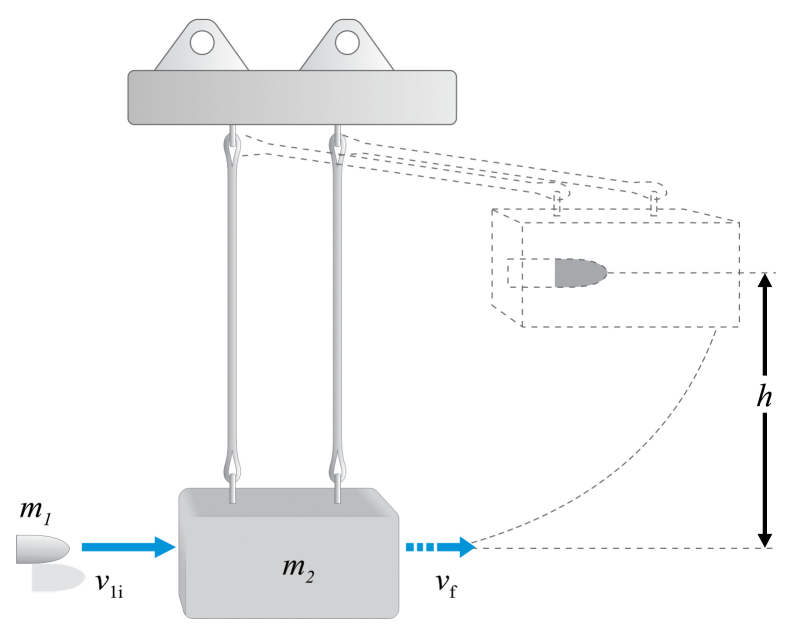

Figura 8: Esquema simplificado de um pêndulo balístico empregado para determinar a velocidade de projéteis.

o que nos permite desprezar essa perda e tratar a colisão como se ela fosse elástica.

Uma aplicação interessante do conceito de colisão elástica é o efeito estilingue gravitacional que sondas espaciais utilizam para elevar sua velocidade e desse modo economizar combustível durante suas viagens. Nessa situação, a interação gravitacional de corpos com massa muito desiguais, por exemplo, um sistema planeta-sonda, conserva o momento linear e, uma vez que a força gravitacional é conservativa, há também conservação da energia mecânica [9]. O uso de assistências gravitacinais, por exemplo, foi empregado durante a viagem da espaçonave International Cometary Explorer $(I C E)$ da NASA. Denominada inicialmente de Sun-Earth Explorer 3 (ISEE-3) e com o objetivo de monitoramento do vento solar entre o Sol e a Terra, a espaçonave ICE foi enviada em uma impressionante jornada de três anos, utilizando assistências gravitacionais em seu caminho até o cometa Giacobini-Zinner conforme pode ser visualizado na figura (9).

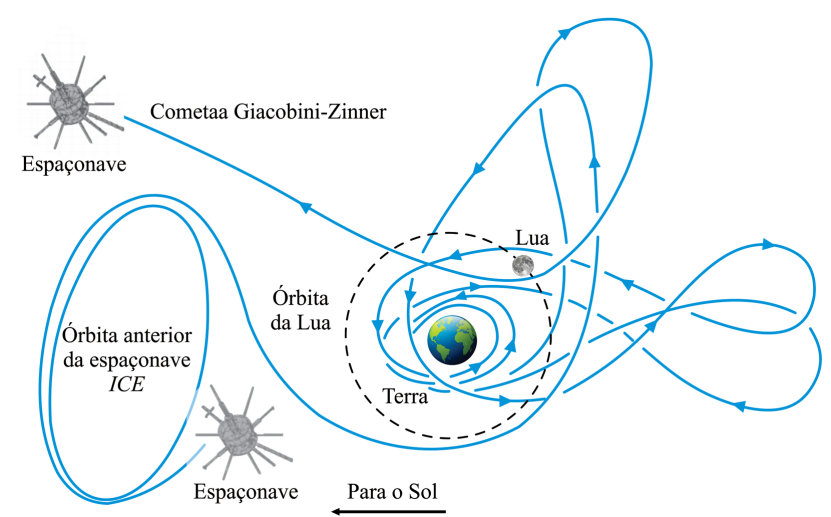

Figura 9: Detalhe da jornada espacial de três anos da ICE até o cometa Giacobini-Zinner, durante a qual, a espaçonave fez uso de assistências gravitacionais [6].

\subsection{Colisões exoérgicas}

As colisões exoérgicas, conforme foi anunciado anteriormente, são caracterizadas pelo aumento da energia cinética do sistema em virtude da diminuição da energia potencial do mesmo. Convém mencionar que é nesse contexto que ocorrem as colisões superelásticas $(e>1)$, cuja maioria de exemplos figura essencialmente na escala atômica, como é o caso da fissão nuclear.

Um exemplo típico de colisões superelásticas é aquelas que ocorrem entre elétrons com átomos ou moléculas excitadas. Em detalhe, quando o elétron colide com uma molécula excitada, esta molécula pode ser desexcitada, transmitindo sua energia de excitação ao elétron que, por sua vez, passa a experimentar um incremento na sua energia cinética. A compreensão dessas colisões permite investigar parâmetros do silício amorfo (a-Si), por exemplo, que é utilizado como camada emissora em células fotovoltaicas.

Nas pesquisas de descargas elétricas em nitrogênio, muitos estudos buscam avaliar os aspectos físico-químicos das colisões eletrônicas superelásticas de desexcitação vibracional das espécies químicas. O interesse pelos fenômenos associados às essas descargas, deve-se ao fato deste gás desempenhar importante papel no tratamento de superfícies em reatores a plasma, em laser a gás e na físico-química da alta atmosfera 10 .

O estudo das colisões superelásticas também é importante no desenvolvimento de tecnologias de microplasmas. Nesse caso, pesquisa-se, por exemplo, as frações de potências transmitidas para elétrons do plasma devido às colisões superelásticas [11]. Os micro-plasmas têm importante aplicações na biomedicina, sistemas de propulsão, controle de fluxo aerodinâmico, processamento de materiais, dentre outros.

Embora, boa parte dos exemplos referentes às colisões exoérgicas ocorram no âmbito microscópico, há casos macroscópicos em que se pode observar tal evento, como é o caso do recuo de uma arma de fogo após o disparo ou a explosão de uma granada.

\section{Considerações Finais}

As classificações das colisões tanto com base na quantidade $Q$ como no coeficiente de restituição são imprescindíveis no estudo da dinâmica de sistemas de partículas. A partir de uma abordagem apropriada foi possível verificar, por exemplo, porque as colisões inelásticas manifestam um coeficiente de restituição entre os casos limites (0 e 1). Isso ratifica ainda o fato que em colisões endoérgicas, o somatório das energias cinéticas das partículas de um sistema após a colisão é sempre menor que o somatório das energias cinéticas das mesmas antes da colisão.

Ademais, pode-se verificar como demonstrar matematicamente a existência de um $e>1$ que, por sua vez, caracteriza as colisões superelásticas ou mesmo as colisões exoérgicas. 
Nesse sentido, a discussão acerca do coeficiente de restituição representa uma importante ferramenta capaz de nos retornar informações pertinentes do processo de colisão.

\section{Agradecimentos}

Enfocamos nossos agradecimentos à profícua parceria entre o Programa de Ciência e Tecnologia da UFOPA e o IFPA, na ausência da qual não seria possível o desenvolvimento do presente trabalho, cujo estímulo decorreu de indagações em plena sala de aula a respeito de como expandir a discussão do coeficiente de restituição para demonstrar a existência de um $e>1$.

\section{Referências}

[1] M.F. Ferreira da Silva, Revista Brasileira do Ensino de Física 30, 1301 (2008).

[2] David Halliday, Robert Resnick e Jean Walker, Fundamentos de Física: Mecânica (LTC, Rio de Janeiro, 2008).

[3] H. Moysés Nussenzveig, Curso de Física Básica: Mecânica (Edgard Blucher, São Paulo, 2002).

[4] J. Barcelos Neto, Mecânica Newtoniana, Lagrangiana e Hamiltoniana (Livraria da Física, São Paulo, 2004).

[5] Hugh Young e Roger Freedman, Física 1: Mecânica (Pearson, São Paulo, 2008).

[6] Stephen T. Thorton e Jerry Marion, Dinâmica Clássica de Partículas e Sistemas (Cengage Learning, São Paulo, 2012).

[7] D.F. Fernandes Marques, Estudo de Balística Interna. Dissertação de Mestrado, Instituto Superior Técnico, Lisboa, 2014.

[8] P. Allen Tipler e Gene Mosca, Física para Cientistas e Engenheiros, Volume 1: Mecânica, Oscilações e Ondas, Termodinâmica (LTC, Rio de Janeiro, 2013).

[9] F.L. da Silveira, L.F.M. Braun e T. Braun, Revista Brasileira do Ensino de Física 32, 3305 (2010).

[10] M.H. Silva Souza, Estudo da Formação de Estados Excitados do Nitrogênio por Impacto de Elétrons. Dissertação de Mestrado, Universidade Federal de Santa Catarina, Florianópolis, 1989.

[11] J.H. Ramalho Gregório, Estudo de Micro-Plasmas Criados por Micro-Ondas a Pressão Atmosférica. Dissertação de Mestrado, Instituto Superior Técnico, Université Paris-Sud (XI), 2010. 